\title{
La flexibilidad laboral en la contratación colectiva en México
}

\author{
Gerardo Tunal Santiago*
}

\begin{abstract}
This article aims to analyze how the collective bargaining came about in the enterprises that placed collective work contracts (CWC) at the Junta Federal de Conciliación y Arbitraje (Federal Meeting of Conciliation and Arbitrage), during the time Zedillo was President, taking as a starting point the political speech on labor flexibility at the beginning of the eighties. Such analysis is done from a reinterpretation, from theoretical and empiric references, of the results obtained from the integral revision of 2,494 CWC that made the Secretaría del Trabajo y Previsión Social (Work and Social Forecast Department) from the 1994-2000 period. Somehow, because of the size of the sample used by such dependency and the temporality reached, there is the possibility of translating the results to assume how the policies were on labor flexibility at the time of collective hiring that were implemented nationally during the time Zedillo was President.
\end{abstract}

Keywords: labor flexibility, collective hiring, productive re-structure, labor regulations, work organization.

\section{Resumen}

Este artículo intenta analizar cómo se dio la negociación colectiva en las empresas que depositaron contratos colectivos de trabajo (ССT) en la Junta Federal de Conciliación y Arbitraje (JFCA) durante el gobierno zedillista, tomando como punto de partida el discurso político sobre flexibilidad laboral a partir del inicio de los años ochenta. Dicho análisis se lleva a cabo a partir de una reinterpretación, a la luz de referentes teóricos y empíricos, de los resultados obtenidos de la revisión integral de 2,494 сCт que hizo la Secretaría del Trabajo y Previsión Social (STPS) durante el periodo 1994-2000. De alguna manera, por el tamaño de la muestra que utilizó dicha dependencia y la temporalidad que abarca, se tiene la posibilidad de trasladar los resultados para inferir cómo eran las políticas sobre flexibilidad laboral en la contratación colectiva que se instrumentaron en el ámbito nacional durante el gobierno zedillista.

Palabras clave: flexibilidad laboral, contratación colectiva, reestructuración productiva, regulación laboral, organización del trabajo.

* Instituto Tecnológico de Estudios Superiores de Monterrey y Universidad Nacional Autónoma de México. Correo-e: gertunsa@yahoo.com.mx 


\section{Introducción ${ }^{1}$}

La incorporación de México al proceso de globalización económica planteó nuevos objetivos de competitividad que obligaron a éste a acelerar sus procesos de reestructuración productiva y a buscar nuevas formas de organización y administración del trabajo. Se trata de desarrollar un profundo proceso de cambio estructural, orientado a recuperar la capacidad del aparato productivo mexicano como sustento del desarrollo económico y social del país. Si bien los retos de la modernidad económica en México se intensificaron en los primeros años de la década de los noventa, es un hecho que éstos no comienzan en 1994 cuando se firmó el Tratado de Libre Comercio (TLC) y se incorporó México a la Organización de Países para la Cooperación y el Desarrollo Económico (OCDE), sino que se iniciaron a mediados de la década de los ochenta, cuando la estrategia de crecimiento económico planteó la necesidad de conformar un escenario macroeconómico sano y estable que apoyara la capacidad de crecimiento productivo. El control inflacionario, el saneamiento de las finanzas públicas y el crecimiento sostenido de las exportaciones -con la modificación sustantiva de sus componentes- nos dan una idea del porqué el aparato productivo mexicano se tiene que ajustar a las nuevas condiciones de globalización económica, por ejemplo vía la flexibilidad laboral. ${ }^{2}$ Particularmente se hace referencia a que el papel que deberán desempeñar los sindicatos mexicanos tendrá que ser diferente al que rigió en el modelo de industrialización por sustitución de importaciones en tanto que, a nivel de discurso, éstos fueron derrotados. Su sustento está sufriendo los efectos de los cambios en el orden internacional, es decir, éstos deberán de integrar los cambios que la puesta en marcha del nuevo modelo de desarrollo ha desencadenado en la economía, la sociedad y el sistema político del país (Zapata, 1995: 159). Dicha integración requiere identificar las nuevas tareas que en términos generales deberán estar orientadas a resolver el problema de representación de las bases en la contratación colectiva, la recuperación del

${ }^{1}$ Una versión preliminar de este artículo fue presentado como ponencia en el XXIII Congreso de la Asociación Latinoamericana de Sociología (ALAS), celebrado en Antigua, Guatemala, en la mesa sobre flexibilidad laboral y cambios en las formas de regulación.

${ }^{2}$ Es importante mencionar que no se trata de llevar a cabo una política laboral de flexibilización salvaje, sino de implantar dicha política en las empresas que presenten características que permitan llevar a cabo dicho proceso y que estén vinculadas a alguna rama económica que tenga un alto impacto en la economía mexicana, y de preparar a las empresas que aún no cuentan con los elementos suficientes para efectuar tal cambio. 
control en los pisos de trabajo, la relación con las gerencias, el rediseño de sus discursos políticos coherentes con las nuevas condiciones económicas y tratar de buscar la articulación con el sistema político que asegure un grado de autonomía mayor que la que tenían hasta los primeros años de la década de los ochenta.

Pese a los mejores esfuerzos y a la buena voluntad de los empresarios, los sindicatos y el Estado mexicano, los ritmos de crecimiento económico aún no se han estabilizado, aunque el reto es todavía el planteado al inicio de los años ochenta, es decir, una tendencia de desarrollo económico continuo. Esta expectativa depende en gran medida de la evolución que se logre en el ámbito de las unidades productivas, las cuales tienen la tarea de aumentar los grados de modernidad laboral para adecuarse a la coyuntura económica actual (globalización económica, apertura comercial, revolución microeconómica, informatización de las organizaciones, etcétera). La adecuación de los procesos productivos a las condiciones citadas líneas arriba ha originado ajustes en la organización del trabajo, pero es importante mencionar que no sólo la flexibilidad laboral y la reestructuración de la fuerza de trabajo son las únicas variables que pueden explicar la integración de las empresas mexicanas al nuevo orden económico y social, sino que hay aspectos en las relaciones laborales, como las formas de supervisión y control sobre el proceso [...] de trabajo [...] las reglas informales y formales de cómo trabajar, las jerarquías de mando, los estilos y niveles de autoridad [...] los problemas del poder, coerción y consenso en la organización (De la Garza, 1998:17), así como las posiciones diferenciales de los trabajadores que en gran medida ayudan a estudiar con mayor objetividad el problema del reto de la modernidad.

Se puede decir que el proceso de flexibilidad laboral, así como el de globalización económica, han evidenciado las condiciones laborales de los trabajadores mexicanos. Una de las particularidades de la flexibilidad laboral es que ésta se presenta como una de las consecuencias más importantes en la reorganización entre capital y trabajo, la cual está produciendo cambios sustanciales en los mercados de trabajo. Grosso modo, se están gestando cambios en la estructura sectorial y ocupacional de la fuerza de trabajo que están modificando las formas de contratación colectiva en México. Por lo anterior, este artículo intenta examinar, a partir de un informe presentado por la Secretaría del Trabajo y Previsión Social (STPS), de qué manera los procesos de flexibilidad laboral impactaron la contratación colectiva negociada du- 
rante el gobierno zedillista y cuáles serían las principales tendencias de dicha contratación en un largo plazo. Se advierte que las afirmaciones expresadas en este artículo son responsabilidad de mi autoría y no reflejan la opinión de la STPS.

\section{Consideraciones metodológicas sobre el informe de la STPS}

Es importante recordar que las reflexiones que en este artículo se hacen provienen de los resultados obtenidos de la revisión integral que hizo la STPS entre los años 1994 y 2000 de los contratos colectivos de trabajo (ССт) reportados en la Junta Federal de Conciliación y Arbitraje (JFCA) durante dicho periodo. Por lo anterior, se hace necesario mencionar los principales criterios del marco muestral utilizados por dicha dependencia para el análisis de los referidos contratos:

i) En el periodo 1994-2000, la JFCA reportó 15,746 revisiones contractuales, en las cuales se beneficiaron a 5,362,999 trabajadores contratados bajo el régimen de contrato colectivo. Dichas revisiones se tomaron como el universo de estudio para el análisis que se realizó. De este universo, la STPS obtuvo una muestra aleatoria irrestricta de 2,494 ССт de jurisdicción federal que incluían a 3'418,390 trabajadores;

ii) Éstos se estratificaron de acuerdo con el número de trabajadores reportados: así, a los contratos con menos de 101 trabajadores se les asignó el estrato 1, mientras que los que reportaron tener entre 101 y 300 trabajadores se les clasificó en el estrato 2, y a los contratos con más de 300 trabajadores en el estrato 3;

iii) Asimismo, la STPS utilizó 114 variables que fueron aplicadas para cada CCT, y

iv) Si bien los ССT de las empresas analizadas fueron los principales instrumentos metodológicos utilizados por la STPS, los reglamentos interiores de trabajo (RIT), los reglamentos de las comisiones mixtas y los convenios sobre productividad también ayudaron en algunos casos a enriquecer los datos obtenidos en la muestra examinada, debido a que en estos documentos, a veces suelen especificarse con mayor detalle algunas prácticas que pudieran inducirnos a analizar con mayor objetividad los avances en cuanto a flexibilidad laboral se refiere; no obstante, es importante mencionar que el acceso a dichos documentos es complicado, ya que no todos se depositan en la JFCA, ni se revisan periódicamente, como sucede con los сст. 
De esta manera, los resultados que se obtienen sobre la flexibilidad en las relaciones colectivas de trabajo pueden estar limitados precisamente por las características de dichos ССT, aunque también pueden ser los instrumentos que nos permitan observar los avances que se han logrado en cuanto a flexibilidad laboral se refiere.

Desafortunadamente, la STPS sólo presentó un análisis de frecuencias excesivamente descriptivo, que se quedó muy corto al tratar de explicar el grado de flexibilidad laboral de los Сст examinados, en tanto que no se les dio un referente teórico a dichos datos ni se realizaron explicaciones del porqué los resultados habían tenido cierto comportamiento; tampoco se presentaron asociaciones entre las variables que pudieran dar cuenta de cómo había sido, es y podría ser en el futuro la contratación colectiva en México. De igual forma, los resultados que obtuvo la STPS sólo fueron utilizados para presentar informes ejecutivos sobre ramas de actividad prioritarias, pero nunca se realizó un análisis global sobre las 35 ramas de actividad económica que tenía tipificadas dicha dependencia.

Por todo lo anterior, me di a la tarea de hacer una revisión exhaustiva de los datos obtenidos por la STPS, con la finalidad de reinterpretar los resultados a través de un referente teórico y de un análisis de asociación que permitiera observar con mayor objetividad y amplitud dicho fenómeno. Particularmente se hizo un seccionamiento por rama de actividad económica, entidad federativa, tamaño de empresa, central obrera y origen del capital, que de algún modo hizo posible relacionar las variables involucradas para ofrecer posibles explicaciones sobre algunas dimensiones metodológicas que puedan medir el grado de flexibilidad laboral clausulado en los ССт de las empresas examinadas por la STPS, así como la posibilidad de analizar dichos datos a la luz de la coyuntura económica y política en la que se fueron negociando éstos, al partir de la evidencia de que no existe un solo tipo de mercado de trabajo, sino una infinidad.

De alguna manera, por el tamaño de muestra que utilizó dicha dependencia y la temporalidad que ésta abarca, se tiene la posibilidad de trasladar los resultados para inferir cómo eran las políticas sobre flexibilidad laboral en la contratación colectiva que se implantaron en el ámbito nacional durante el gobierno zedillista.

Sin olvidar que existen otros parámetros que miden la flexibilidad laboral, en este análisis se hace hincapié en indicadores 
orientados a la aplicación de la flexibilidad numérica, la flexibilidad funcional, la participación de los trabajadores en la organización del trabajo, la capacitación y su bienestar. ${ }^{3}$ Se advierte que las aseveraciones presentadas sólo hacen referencia a la parte formal de las empresas ${ }^{4}$ y por lo tanto no pueden ser extensivas a las prácticas informales llevadas a cabo en el interior de éstas.

\section{Los estudios sobre la flexibilidad laboral}

Es un hecho que la mayoría de los estudios sobre flexibilidad laboral, no sólo en México sino en muchos países, se han orientado a presentar investigaciones cuyo objeto de estudio son las empresas que aparentemente tienen gran impacto en la economía. Lo anterior ha provocado, por lo menos en México, un abuso del estudio de esa parte de la realidad y el consecuente rezago de algunas disciplinas que han intentado abordar dicha problemática. El nuevo orden internacional no sólo ha cambiado a las economías, sino que ha desencadenado nuevas situaciones que generaron nuevas formas de producir y, consecuentemente, nuevos tipos de negociación laboral.

Si bien es cierto que todavía hace algunos años ciertos sectores económicos se vislumbraban como la punta del desarrollo y de la integración de México al primer mundo, también es un hecho que la realidad ha cambiado, rebasando los supuestos que

\footnotetext{
${ }^{3}$ Los tres primeros los agrupo en la dimensión metodológica que denomino organización productiva, mientras que los dos últimos los ubico en la dimensión nombrada como recursos humanos.

${ }^{4}$ Se consideran como prácticas formales las acciones negociadas entre sindicato y empresa y que quedan estipuladas en un convenio de carácter legal. Es importante mencionar que las relaciones laborales no sólo pueden explicarse a través de los clausulados de los СCT y/o RIT, en tanto que existen prácticas en el interior de los pisos de trabajos que tienen su dinámica por los usos y costumbres derivados de las subjetividades de la relación diaria entre trabajadores y gerencias, y en donde casi siempre los cambios en la organización del trabajo se deciden unilateralmente por las primeras y son presentadas al sindicato pero sin posibilidad de objeción. De igual forma, es importante mencionar que en muchos casos el grado de flexibilidad de los сст no es visible, debido a que las formas de pago y contratación se realizan en los hechos y no se registran en dichos contratos, como ocurre en algunos casos con el pago de bono de productividad y con la contratación de trabajadores de confianza; incluso un gran peso de la muestra fue ubicado en el rubro de respuestas contestadas como no especificadas. Tentativamente, se puede sugerir que lo anterior se debe a que los cambios sustanciales en las condiciones generales de trabajo y las decisiones con respecto a éstos son pactados y se dirimen, en algunas empresas, en el espacio de la informalidad del trabajo. Aunque, finalmente la reestructuración productiva que está experimentando nuestro país, en una de sus vertientes, está dirigida precisamente a disminuir estos usos y costumbres a través de la codificación de todos los aspectos derivados de la relación entre capital y trabajo (De la Garza, 1999).
} 
muchos científicos sociales se habían hecho con respecto a esta situación. Los estudios de la flexibilidad laboral de las maquiladoras, sector eléctrico, empresas dedicadas a la elaboración de bebidas, industria azucarera, minería, ramas dedicadas a la metalúrgica y siderúrgica, petroquímicas, industria automotriz y de autopartes, ferrocarrileros, servicios de banca y crédito, trabajadores del transporte aéreo, telefonistas, servicios educativos y de investigación, podrían ya no ser tan pertinentes para la sociología del trabajo, en tanto que las formas de trabajo que hasta hace poco se consideraban atípicas, hoy en día forman parte de la nueva realidad en algunos países.

Luego entonces, los estudios laborales del futuro próximo deberán estar orientados a estas nuevas realidades en las que el trabajo asalariado va perdiendo fuerza. Si se piensa en algunos países de América Latina, el sector informal ha crecido y se ha a convertido en la realidad laboral de la región, lo que plantea nuevas situaciones en las que la flexibilidad laboral toma otro rumbo. ${ }^{5}$ Asimismo el abuso de los estudios sobre ramas prioritarias se convierte en un sesgo para el análisis de los fenómenos laborales en México, en la medida en que olvida que la realidad laboral y productiva de este país no sólo se vincula con las maquiladoras de la frontera norte, ni con la tecnología de los servicios telefónicos, sino que hay un abanico inmenso de realidades laborales que se ha olvidado estudiar, por ejemplo las industrias cinematográfica, del transporte marítimo, del sector hulero, de la rama dedicada a los hidrocarburos, cementeras, caleras, químico-farmacéutica, del ramo de la celulosa y papel, productoras de alimentos, del sector de los aceites y de las grasas vegetales, de las madereras, vidrieras, tabacaleras, del transporte terrestre, de la construcción, de la asistencia médica y social, de la radio y la televisión, y de la distribución de gas, entre otras.

\footnotetext{
${ }^{5} \mathrm{Al}$ hablar del sector informal es importante mencionar que se trata de un gran sector que está sustentado por una producción marginal de pequeña escala que está limitada por el uso de tecnologías simples, pequeño capital y falta de relaciones con otros sectores. Según Meyer (1989), el sector informal está caracterizado por: i) fácil entrada; ii) confianza en recursos indígenas; iii) relaciones de trabajo sustentadas por relaciones de tipo familiar; iv) pequeñas escalas de operación; v) trabajo intensivo y adaptación tecnológica; vi) calificaciones adquiridas fuera de la educación formal, y vii) desregulación y competitividad de los mercados. Asimismo, en el sector informal urbano existe mayor flexibilidad y menos influencia de las variaciones en los suministros producidas por la demanda (Wong, 1996). En términos generales se puede decir que el sector informal está referido a un conjunto de unidades productivas de baja capitalización que se asocia con el uso de tecnologías obsoletas de bajo costo.
} 
También resulta importante mencionar que muchos de los estudios sobre flexibilidad laboral, surgidos en la llamada sociología del trabajo, han explicado el fenómeno de la flexibilidad laboral sin darle un referente histórico y sin vincularlo seriamente con la cuestión sindical. Muchas veces se ha tratado de trabajos que sólo pueden discutirse de manera abstracta en la academia o entre los grupos intelectuales dedicados al estudio de dicho fenómeno. Estas investigaciones casi nunca han planteado soluciones que puedan traducirse en política laboral; por el contrario, han olvidado que el fenómeno de la flexibilidad es un fenómeno de la realidad, que si bien puede ser analizado en el ámbito de las teorías, es un hecho que no podemos desprenderlo definitivamente del plano de lo real. Otra de las grandes deficiencias en los estudios sobre la flexibilidad laboral radica en que se ha cometido un exceso al analizar esta problemática desde la economía del trabajo, cuando el problema de la flexibilidad laboral se ha gestado precisamente vía la contratación colectiva, de manera que también se ha hecho a un lado toda esa parte histórica sindical que contextualizó y determinó la contratación colectiva en México. En términos generales, muchos de los estudios sobre flexibilidad laboral, vistos desde la sociología del trabajo, no han caído en la cuenta de que las estrategias de flexibilidad laboral dependen no sólo de las características de cada país, sino también del tipo particular de sector económico y de empresa que se esté analizando.

Los cambios que ha sufrido el mundo en las últimas dos décadas se han direccionado hacia muchos niveles y éstos han sido tan radicales que resulta una batalla perdida intentar abarcarlos a partir de un nuevo monismo teórico. La política cambió, las economías nacionales se globalizaron, los sistemas productivos se vincularon a la tecnología, los mercados de trabajo son otros, la organización del trabajo reconoce nuevas realidades laborales, la fuerza de trabajo es otra, las relaciones laborales se negocian de manera distinta, los sistemas de relaciones industriales toman otro rumbo, los pactos corporativos se están fracturando, la identidad de los trabajadores en sus lugares de trabajo es otra, las organizaciones clasistas están cambiando, las subjetividades laborales son distintas, y el fantasma de la flexibilidad laboral comienza a esparcirse en los pisos de trabajo. Grosso modo, los paradigmas teóricos que dominaron casi todo el siglo xx han dejado de ser pertinentes. Se habla entonces no de una simple crisis económica, sino de todo un proceso de transformación que 
para su explicación requiere de una perspectiva integral de todas las ciencias de lo social.

Ahora los cambios en la organización del trabajo ya no pueden ser analizados partiendo del monismo de una función de producción determinada por variables económicas, sino del supuesto de que la producción implica una relación social que no sólo abarca los pisos de trabajo, sino que afecta la organización social en su conjunto y produce cambios sociales que van desde la identidad de los actores sociales hasta las políticas de tipo gubernamental. De alguna forma, es hasta con las perspectivas japonesas del management cuando se reconoce que el punto de análisis implica la trayectoria de los costos hasta la participación y el involucramiento de los trabajadores en el proceso de trabajo, utilizando la ecuación de calidad total, justo a tiempo y consenso.

Indudablemente hasta los primeros años de la década de los ochenta la discusión teórica que daba cuenta de la realidad latinoamericana era muy amplia en tanto que incluía conceptos que vinculaban los aspectos políticos, económicos y sociales. Se hablaba, por ejemplo, de formación socioeconómica, industrialización sustitutiva, relación del desarrollo $v s$. subdesarrollo, clases sociales, patrón de acumulación, economía, Estado, etcétera. Pero es también un hecho que a partir de la crisis del modelo de sustitución de importaciones, dicho pensamiento también entró en crisis, de manera que éste no encontró acomodo en las condiciones que se estaban presentando en el mundo y provocó una ruptura histórica de las ciencias de lo social, la cual se produjo en pro de perspectivas reduccionistas que argumentaban que la parte cultural no tenía un vínculo estrecho con la cuestión política, de tal forma que dichas teorizaciones de la realidad suponían que el mercado subsume a la sociedad y a la política asintóticamente y las variables no económicas aparecen a lo sumo como parte de un residuo impreciso por las dificultades de matematizarlas (De la Garza, 1999: 3). Indudablemente es un hecho que en otras coyunturas el monismo reduccionista de la teoría no tuvo mucha aceptación, sino que por el contrario se pensaba en explicaciones diversificadas que coexistían con otras visiones más culturalistas (toyotismo, ${ }^{6}$ especialización flexible, segmentacionismo, regulacionismo, lean production, etcétera).

\footnotetext{
${ }^{6}$ El toyotismo es una 'filosofía gerencial' de organización del trabajo en la que se contempla la gran crisis capitalista y la salida de ésta a través de la experiencia japonesa que data desde la década de los años cincuenta y que de alguna manera fue revoluciona-
} 
Por lo anterior los estudios actuales de la flexibilidad laboral, si bien no deberán obviar que la contratación colectiva implica una optimización de costos, tampoco deberían obviar que no hay una interacción social, de tal suerte que los conceptos que involucren a la cultura en un sentido amplio deberán utilizarse con mayor frecuencia para explicar el fenómeno de la flexibilidad laboral en la contratación colectiva (competitividad empresarial, relaciones inmediatas de las empresas con su entorno, tecnología, relaciones laborales, características de la fuerza de trabajo, productividad, calidad y política, entre otras).

\section{La flexibilidad laboral en México}

\section{Al finalizar el fordismo ${ }^{7}$ como paradigma dominante del capita- lismo contemporáneo, los sistemas de relaciones industriales se}

da en la década de los ochenta. Por un lado, en esta doctrina se hace una crítica a la rigidez taylorista de escindir abruptamente la concepción y la ejecución del trabajo. Por el otro lado se plantea una organización del trabajo sustentada en los conceptos de calidad total, justo a tiempo y consenso. Se trata de una nueva actitud del trabajador hacia su trabajo, y particularmente se hace una valorización cualitativa de los productos que sustituye al valor de cambio. Lo anterior implicó un cuidado especial de los aspectos culturales de los trabajadores, de manera que las gerencias empezaron a tener dentro de sus programas de trabajo apartados que tenían que ver, por ejemplo, con la identidad de los trabajadores, con su centro de trabajo y con los productos generados por éstos. En el toyotismo se trata de recuperar el saber hacer obrero y de involucrar a los trabajadores en los procesos de trabajo vía la participación, capacitación integral y la reintegración de labores. En este modelo se plantea la necesidad de implantar una flexibilidad productiva consensuada en donde se le dé mayor énfasis al mercado interno en vez del mercado externo, de modo que se trata de una flexibilidad interna, es decir entre puestos, áreas, turnos y horarios de trabajo, ascensos en función de la capacidad y no por antigüedad, y salarios de acuerdo con el desempeño de los trabajadores. Luego entonces, no se trata de una flexibilidad externa en la que el empleo y el desempleo queden vinculados con las necesidades de la producción. Una de las cuestiones más interesantes de esta filosofía es que se trata de flexibilizar los procesos de trabajo en vez de desregular los mercados de trabajo y de reconocer que los sindicatos no son una rigidez que se tenga que minimizar. De igual forma, no se busca el equilibrio, sino mejorar continuamente. En términos generales se puede decir que en el toyotismo la idea del individualismo y la concepción del actor racional fue sustituida por los conceptos de trabajo en equipo, mejora continua, cero errores, círculos de calidad, flexibilidad, polivalencia, cultura laboral, relación cliente-proveedor, empresa como comunidad, y acción de acuerdo con valores y no sólo con utilidad.

${ }^{7}$ El llamado periodo fordista no sólo fue la aplicación de un esquema técnico o gerencial para la organización del trabajo. Se trataba también de un patrón de acumulación de capital sustentado en una ampliación sin precedentes del consumo masivo y una forma particular de organización del trabajo. Dicho modelo de acumulación se empezó a dar en la segunda década del siglo xx en las industrias estadounidenses, aunque al final de la Segunda Guerra Mundial comenzó a extenderse a las industrias de los principales países capitalistas, obviamente con matices muy diferentes, pero que fue el marco regulatorio de la economía en su conjunto. En términos generales, dicho paradigma productivo puede entenderse a través de: i) la división técnica del trabajo establecida en el taylorismo, la cual implicó la producción masiva de bienes estandarizados, la aplicación de tecnologías rígidas vinculadas a las cadenas de montaje y a una fuerza de trabajo especializada; ii) el incremento de la productividad sustentado en la mecanización e 
vieron en la necesidad de buscar e implantar nuevas estrategias productivas que hicieran frente a la nueva coyuntura económica que se estaba gestando en el orden internacional. En el terreno de la producción, la dinámica de la flexibilidad empezó a sustituir a las economías de escala; en el terreno de la regulación estatal, las políticas de control monetario limitaron la aplicación del gasto presupuestal como instrumento de estímulo al consumo, en medio de un desdoblamiento generalizado en la producción de bienes colectivos y servicios; en cuanto al consumo, comenzó a presentarse una rápida diversificación de los mercados que en consecuencia motivó la innovación en el diseño de productos y la variedad, tanto en la producción en serie como en la de pequeños lotes.

De alguna forma resulta interesante plantearse si los modelos de organización del trabajo se dirigen hacia la flexibilidad (posfordismo, ${ }^{8}$ lean production, especialización flexible), o si siguen la trayectoria de la lógica de etapas y funcionalidades pro-

intensificación del trabajo, y iii) un incremento del consumo basado en la estabilidad en el empleo, el crecimiento de los salarios reales y una cobertura amplia de seguridad social. El modelo fordista fue puesto a andar a través de un marco de crecimiento y estabilidad que no fue capaz de mantenerse como paradigma articulador en una coyuntura de incertidumbre económica y consecuentemente de mercados poco estables. Lo anterior produjo durante la década de los ochenta y los noventa un dificultoso proceso de reestructuración económica, que con distintas graduaciones y ritmos involucró a la economía mundial.

${ }^{8}$ A finales de la década de los años sesenta empezó a cuestionarse el sistema fordista a medida que las relaciones sociales eran cada vez más tensas y los antiguos acuerdos en torno al pleno empleo y a la financiación de un Estado del bienestar cada vez más caro se debilitaban, presionando a los gobiernos. La crisis del fordismo ha impulsado a muchos analistas a defender que el desarrollo del capitalismo de mercado ha generado, de hecho, un sistema de producción y de relaciones sociales posfordista. El capitalismo posfordista se caracteriza por la desaparición de algunas de las características de su antecesor; los métodos de producción se centran ahora en nuevas tecnologías productivas, como la biotecnología, pero sobre todo la microelectrónica, y la tecnología de la información. Además, las relaciones y prácticas laborales posfordistas son más flexibles, como muestran las relaciones laborales de las corporaciones japonesas. El keynesianismo fue perdiendo importancia a medida que el monetarismo (que se caracteriza por su fe ciega en las fuerzas del mercado para alcanzar objetivos económicos) hegemonizaba la ciencia económica. Un nuevo individualismo reemplazó la confianza anterior en las instituciones colectivistas del fordismo. Al tiempo que se producían estos cambios, los sindicatos iban perdiendo fuerza (y afiliados), lo que les forzó a aceptar un 'nuevo realismo' sobre su papel en la sociedad; asimismo, se reducía la intervención del Estado, como se demuestra por el creciente número de privatizaciones realizadas en todos los países con economías de libre mercado. Sin embargo, hay que destacar que sigue existiendo una importante polémica en el plano académico sobre las características y los efectos de las instituciones de la era posfordista, lo que sin duda es una consecuencia de la cantidad de instituciones existentes en las economías capitalistas y las diferencias que existen entre éstas. Es interesante mencionar que las corrientes que se agrupan en el postfordismo (regulacionismo, especialización flexible y neoshumpeterianismo) convergen en que la producción en masa estándar está agotada y que se está transitando hacia nuevos paradigmas productivos flexibilizados (De la Garza, 1999). 
pias de los modelos teóricos de corte regulacionista (taylorismo, ${ }^{9}$ fordismo, etcétera). Aquí es importante hacer un alto para aclarar que, pese a lo que argumentan los modelos funcionalistas, la discontinuidad, contradicción y disfuncionalidad no siempre significan anormalidad. Se estaría más de acuerdo con los paradigmas no funcionalistas que plantean que las estructuras son coerciones no determinantes sobre los actores sociales, de tal modo que las respuestas de la realidad están sustentadas sí por dichas estructuras, pero también por la interacción social que se da al interior de éstas. Obviamente que lo anterior nos conduce a pensar en procesos en los que no solamente están involucrados factores productivos mundiales y racionalidades universales que explican el ocaso del taylorismo y del fordismo, sino en la noción de contexto y de convergencia de organizaciones de trabajo que coexisten de forma diferenciada.

En este contexto de globalización económica, México tuvo que buscar también nuevas salidas que hicieran frente a la crisis del patrón llamado de sustitución de importaciones que se evidenció en los años de $1982^{10}$ y 1995 . Frente a este nuevo panora-

${ }^{9}$ El taylorismo, basado en el principio de la 'administración científica' desarrollado por Frederick Winslow Taylor, puede considerarse como la racionalización de los procesos productivos al diferenciar las tareas de creación y ejecución, o lo que es lo mismo, al dividir la organización de la producción (directivos, ingenieros, entre otros) de las tareas mecánicas, asegurando un mayor control de la producción por parte de los gestores. Así, los aspectos mentales quedan separados por completo de las tareas manuales. Esto constituyó una ruptura total con los métodos de producción del pasado, cuando la producción se organizaba en función del tipo de artesanía y los artesanos creaban, organizaban y completaban las tareas manuales. Los movimientos sindicalistas se opusieron a los cambios que implicaba el taylorismo, pero los aceptaron a cambio de recibir un mayor porcentaje de las ganancias de productividad derivadas de la racionalización e intensificación de los procesos productivos. Este compromiso sólo fue aceptado por un pequeño número de empresarios (entre ellos Henry Ford) y a pesar de la defensa de este acuerdo que realizaron grandes economistas, como John Maynard Keynes, sólo tras la II Guerra Mundial se aceptó de un modo general el acuerdo entre empresarios y trabajadores. El aparente buen funcionamiento del taylorismo se debió a la existencia de una serie de instituciones -como el colectivismo de las relaciones laborales, una especie de Estado del bienestar que garantizaba unos niveles de vida mínimos de forma que, aunque algunos agentes no fueran activos desde un punto de vista económico (como los jubilados o los desempleados, entre otros), todos tenían una capacidad de consumo- y al desarrollo de los modernos sistemas crediticios y bancarios. Todo ello permitía al Estado desempeñar un papel activo en la gestión de la economía, tanto de forma directa, utilizando el gasto público (mediante las políticas de demanda keynesianas), como por vías indirectas al regular el sistema crediticio. A escala internacional, la coordinación y el comercio entre países desarrollados (que aplican el sistema de producción fordista) creció bajo la hegemonía de Estados Unidos, interesados en evitar la expansión del comunismo, para lo que invirtieron enormes sumas (como el Plan Marshall) asegurando la adopción del sistema fordista en los países de Europa y Oriente.

${ }^{10}$ La crisis del modelo de sustitución de importaciones se evidenció primeramente porque el sector agrario dejó de generar divisas, en tanto que el índice de exportaciones decreció y se dejaron de vender insumos y alimentos a precios accesibles. Lo anterior provocó un intercambio desigual con los sectores industriales. Para 1981 la caída de las 
ma, en México se hacía necesaria una reestructuración económica que se sustentara en una flexibilidad consensuada del tipo de los modelos toyotistas y en la cual se involucrara al Estado, las cúpulas sindicales, las gerencias y en gran medida a los trabajadores (Castilla y Torres, 1999). Las opciones no eran muchas ya que los modelos económicos emergentes y con mayores posibilidades tenían que ver con los paradigmas productivos implantados en y para los países del primer mundo. A este respecto W. Gunter Remmling argumenta que los paradigmas teóricos del mundo occidental se presentan como universales, pero que en la realidad fueron, a pesar de su aspiración expresa, mucho menos universales de lo que habitualmente se supone (Remmling, 1990: 69 ), en tanto que el mundo actual se caracteriza por realidades fragmentadas, en donde no es posible establecer racionalidades universales. De igual manera se puede plantear que muchas de las discusiones teóricas actuales sobre la organización del trabajo llevan implícitos elementos utópicos que han implicado el dominio de éstas, en tanto que se presentaron como una opción a los modelos de acumulación taylorista y fordista, aunque realmente se trataba de etapas incipientes que tuvieron la posibilidad de extensión y profundización a través de la concientización de las gerencias y de los trabajadores.

Es así como los sistemas de relaciones industriales en México empezaron a contemplar la nueva cultura laboral que propagaba el toyotismo, el fujitsuismo, el posfordismo. Esta referencia conceptual responde precisamente a que en México la coyuntura económica, política y social es distinta a la de las naciones industrializadas y por lo tanto la discusión en torno a la organización del trabajo tiene que adecuar algunos elementos de dichos paradigmas que en el plano de lo real sean aplicables y explicables de las empresas mexicanas. Se trata no sólo de rebasar los reduccionismos teóricos desde las experiencias de los países industrializados, sino también de la producción de paradigmas teóricos y políticas laborales concretas que sean pertinentes para los contextos en los que dicho fenómeno esté surgiendo o se esté implantando. El proceso no ha sido fácil y no sólo por la resistencia de los actores sociales involucrados, sino por las características propias de la coyuntura actual que se vive en México. Frente a la situa-

tasas del crudo y el incremento de las tasas internacionales de interés provocaron un funcionamiento deficitario del gasto social. Indudablemente que esta crisis de modelo productivo se presentó como una crisis agraria y financiera que originó el rediseño de la política estatal. 
ción anterior, México ha tratado de implantar un toyotismo a la mexicana o una flexibilidad primitiva (De la Garza 1999; Bouzas y De la Garza, 1999) proceso en el que uno de los retos principales es flexibilizar los procesos de trabajo y revalorizar el trabajo a través de la presencia de equipos de producción con participación activa de los trabajadores y del sindicato en el incremento de la productividad y el pago de ésta (Castilla y Torres, 1999: 586).

\section{La contratación colectiva en las empresas mexicanas}

El análisis de la flexibilidad de las condiciones generales de trabajo presenta algunas limitaciones producto de las características del desarrollo de las relaciones de trabajo colectivas en México de modo que:

i) La contratación colectiva se refiere a convenios que realizan trabajadores y empresarios para legitimar relaciones de trabajo del personal sindicalizado y que el CCT es el documento legal en donde se estipulan, como mínimo, las condiciones laborales que garantiza el Artículo 123 constitucional y la Ley Federal del Trabajo (LFT) y puede ser independiente a otro tipo de convenios;

ii) Las relaciones colectivas de trabajo en México han estado sustentadas en la representación corporativa de obreros y empresarios, cuyas cúpulas pactan de manera general las condiciones obrero-patronales de una gran diversidad de actividades económicas como si la firma de estos pactos sólo fuera un requisito legal o administrativo, por lo que en ocasiones no correspondan a la especificidad de una empresa en particular;

iii) Si bien en la mayoría de los ССт hay una gran ausencia de especificaciones, por omisión se sugiere que podría existir un margen grande de unilateralidad por parte de las gerencias para decidir sobre los cambios en las condiciones generales de trabajo. Aquí es pertinente recordar el argumento que hace Enrique de la Garza en cuanto a que las tendencias a obedecer las instrucciones de los líderes sindicales ha obstruido la participación de las bases en la organización del trabajo; obviamente lo anterior ha sido avalado por las gerencias y ha provocado decisiones unilaterales en cuanto a los cambios en los procesos de trabajo;

iv) Tradicionalmente los СCT se concentran en cláusulas sobre salarios, prestaciones y en acuerdos sobre la jornada de trabajo, con lo que quedan poco reglamentados los aspectos que tienen que ver con la organización del trabajo, y 
v) El сCт es el único acuerdo de negociación colectiva que puede invocarse para que estalle una huelga.

Como un intento de darle fin a la crisis productiva iniciada a mediados de la década de los años sesenta, los sistemas de relaciones industriales propusieron algunas formas de organización productiva que flexibilizarían, entre otras cosas, las condiciones generales de trabajo. De una u otra forma dichas propuestas incluían la necesidad de que los empresarios tuvieran la capacidad de contratar, despedir y reubicar mano de obra, así como de hacer partícipe a ésta en los cambios de los procesos de trabajo. Casi al finalizar el siglo xx la organización productiva del trabajo, junto con otros factores, se convirtió en un aspecto esencial para señalar la eficiencia productiva de las empresas. Particularmente la flexibilidad numérica y funcional, así como la firma de pactos bilaterales entre empresarios y sindicatos para tomar decisiones en la organización del trabajo, se convirtió en el eje para analizar la capacidad que tienen las empresas para flexibilizar sus condiciones generales de trabajo. En términos generales se trataba de una nueva forma de organización productiva que tendería a la flexibilización y/o desregulación laboral.

Antes de hablar de este tipo particular de flexibilidad, se hace necesario mencionar que "[...] la flexibilidad del trabajo [...] es entendida como [...] la capacidad [...] que tiene [...] la gerencia de ajustar el empleo, el uso de la fuerza de trabajo en el proceso productivo y el salario a las condiciones cambiantes de la producción" (Bouzas y De la Garza, 1999: 44). Asimismo, este concepto hace referencia al conjunto de dispositivos puestos en práctica por una organización productiva que responde a la creciente variabilidad de productos, mercados, diversificación de los procesos de producción y de las relaciones laborales (Trouve, 1989). Desde la década de los años ochenta la noción de flexibilidad se convirtió en la palabra clave para comprender los cambios que se estaban gestando en las relaciones laborales. Al concepto anterior se le vinculó con:

i) La tecnología reprogramable;

ii) Las nuevas formas de organización del trabajo que le darían salida al desgaste de los modelos taylorista y fordista;

iii) La precariedad en los empleos;

iv) Las transformaciones de los sistemas de seguridad social, de la contratación colectiva y de las leyes laborales, y

v) La ruptura de los pactos corporativos en el ámbito estatal. 
Por lo anterior, resulta importante aseverar que la noción de flexibilidad laboral no tiene un significado único, debido a que en México las formas y contenidos de ésta tienen que ver con las diferentes estrategias empresariales de modernización, y de ahí que se hable de flexibilidad numérica, funcional o salarial. Entonces la flexibilidad laboral no es un simple asunto de técnica económica o administrativa, es sobre todo una materia políticosindical y, por tanto, no sigue caminos lineales, depende de relaciones de fuerzas, de concepciones, de capacidad de negociación y de propuestas, a pesar del discurso dominante que deslegitima la presentación de alternativas y considera como algo natural a la flexibilidad (Bouzas y De la Garza, 1999: 77-78).

La flexibilidad numérica de personal, entendida como el incremento de la cuota de capital variable movilizado en el proceso productivo -no sólo por la tecnificación de éste, sino también por una mejor organización del mismo- expresa la capacidad de la empresa para modificar la plantilla de trabajadores en función de las variaciones de la demanda externa de sus productos (González, 1999: 349). Así también, la flexibilidad numérica es un indicador que nos ayuda a entender la capacidad que muestran las empresas para adecuar sus condiciones generales de trabajo al proceso de modernidad productiva. Las variables utilizadas para medir el indicador que ahora nos ocupa fueron las siguientes: el ingreso de nuevos trabajadores, la separación de éstos de sus centros de trabajo, el tiempo de trabajo y la contratación de trabajo eventual o de mano de obra para trabajos fuera de la operación normal. De los CCT examinados se puede observar lo siguiente:

i) Pese a que el ingreso de nuevos trabajadores es una decisión bilateral entre empresa y sindicato $(80.97 \%)$ y el periodo de prueba para dichos trabajadores es una constante (61.69\%), la evaluación del trabajador de nuevo ingreso en el periodo de prueba es todavía una decisión unilateral (89.73\%);

ii) Las horas de trabajo por semana siguen estando por arriba de las 45 horas (80.34\%), mientras que la duración de las jornadas diurna, nocturna y mixta se ubican por arriba de las 40 , 35 y 37.5 (98.63\%, 99.17\%, 98.75\%, respectivamente);

iii) El número de turnos en operación sigue estando por debajo de 4 (95.24\%);

iv) En promedio los días de vacaciones al año de labores se ubican por debajo de los 9 días (77.97\%), y 
v) Pese a que la propuesta de candidatos para contratar personal eventual o temporal, en un gran porcentaje es producto de una decisión bilateral, ya sea de la gerencia o del sindicato (86.63\%), la contratación de algún trabajo especial no es una decisión en la que se consulte a los sindicatos (34.24\%) o en el peor de los casos, ni si quiera existe esta posibilidad (56.95\%).

De manera más explícita se puede observar que la propuesta de personal por parte de los sindicatos para este tipo de trabajos es casi nula (77.95\%). En los ССт examinados se observa un incremento de la flexibilidad numérica de 6.78 puntos porcentuales a una tasa media de crecimiento anual (TMCA) de $2.56 \%$, lo cual podría reflejar que el avance de los contratos analizados aún es lento. Lo anterior puede reforzarse con los datos obtenidos al hacer un seccionamiento por ramas de actividad económica en el que se observa que 17 de éstas obtuvieron una flexibilidad numérica promedio menor a la media general. Indudablemente existen muchas ramas económicas que muestran excesiva rigidez y en consecuencia rezago en sus procesos de contratación de mano de obra; tal es el caso de las empresas dedicadas a la industria de aceites y grasas vegetales, transporte terrestre y cementera, que en alguna forma están impactando fuertemente a la media general en este rubro. Al examinar la flexibilidad numérica del personal clasificada por estrato de trabajadores, se observó el siguiente comportamiento: el 69.49\% de los contratos (los de los estratos 2 y 3) reportaron una flexibilidad numérica promedio mayor a la media general, mientras que las empresas clasificadas en el estrato $1(30.51 \%)$ están por abajo de la media, en 6.02 y 8.80 puntos porcentuales.

Lo resultados anteriores nos indican que el contenido de los CCT y RIT examinados, no contemplan en su mayoría suficientes cláusulas que permitan medir o indicar que se están aplicando políticas orientadas a poner en práctica la flexibilidad numérica de sus trabajadores, de modo que se puede sugerir que la flexibilidad numérica del personal amparado por dichas negociaciones aún no es una política que estén instrumentando los empresarios de manera generalizada en los centros de trabajo. ${ }^{11}$ Posiblemente

\footnotetext{
${ }^{11}$ Aunque hay que reconocer que algunas empresas grandes presentan la tendencia de procesos productivos que obligan a trabajar los siete días de la semana, en varios turnos, lo que se acompaña de una mayor intensificación de los ritmos de las tareas y de cambios importantes en lo que se refiere a jornada de trabajo, horarios, días de descanso obligatorios, vacaciones, ascenso y movilidad horizontal, y que en términos generales se entiende como flexibilidad numérica.
} 
se pueda sugerir que la resistencia de los trabajadores aglutinados en centrales obreras fuertes no ha permitido lo anterior, ya que para éstos la flexibilidad numérica podría significar vulnerabilidad en sus puestos de trabajo.

Otro de los indicadores que sirve para medir el grado de flexibilidad en las condiciones generales de trabajo es la llamada flexibilidad funcional de personal, la cual nos señala el uso flexible de la fuerza de trabajo dentro de los procesos productivos, lo que implica generalmente un trabajador polivalente, ${ }^{12}$ así como el establecimiento de movilidad interna entre los puestos de trabajo, departamentos, adscripción, turnos y horarios, entre otras. La flexibilidad funcional se refiere a la capacidad de la empresa para modificar la estructura de puestos de trabajo y reasignar las tareas de los trabajadores en función del cambio tecnológico (González, 1999: 349). Las principales variables que se utilizan para entender este indicador tienen que ver con la promoción a puestos de trabajo superiores, los cambios de puestos y funciones, las modificaciones de horarios o días de trabajo y los sistemas de trabajo o maquinaria.

En el periodo analizado, el indicador que se está examinando presentó un incremento de 2.10 puntos porcentuales que equivalen a una TMCA de 0.81 por ciento. El dato anterior se debe a que la flexibilidad funcional promedio para 1996 cayó 6.53 puntos porcentuales con respecto al dato anual de 1995. De igual forma, para 1997 la flexibilidad funcional promedio todavía fue inferior a los niveles de 1994 y 1995. Los resultados anteriores nos podrían dar evidencia para suponer que la flexibilidad funcional de personal es todavía una tarea difícil con la que se enfrentan las empresas observadas. Por ramas de actividad se puede ver que poco más de la mitad de éstas (58.62\%) obtuvieron una flexibilidad funcional superior a la media. Los datos anteriores podrían indicar que algunas ramas de actividad económica que tuvieron un alto índice de flexibilidad funcional ${ }^{13}$ producen resultados favorables en este rubro. En cuanto al tamaño de las empresas, se observa que sólo los contratos con más de 300 trabajadores rebasan la media general de flexibilidad funcional obtenida. Probablemente las empresas clasificadas en el estrato $1 \mathrm{y}$ 2 no cuentan con trabajadores polivalentes y la movilidad en el

\footnotetext{
${ }^{12}$ Aquel que puede ser destinado a tareas diferentes de aquellas para las que fue contratado, con lo cual adquiere mejores habilidades.

${ }^{13}$ Por ejemplo las empresas de las ramas calera, ferrocarrilera, eléctrica y cinematográfica.
} 
interior de éstas podría estar muy restringida. Una vista más detallada de este fenómeno se refleja en los siguientes datos:

i) De los contratos examinados que presentan cláusulas sobre quién toma la decisión para ascender a los trabajadores a puestos superiores, $51.41 \%$ indica que se trata de una decisión bilateral. La promoción de los trabajadores a puestos superiores no es todavía un proceso integral, ya que los indicadores que permiten observar la medida en que los contratos colectivos incluyen aplicación de políticas de flexibilidad funcional aún presentan niveles bajos: ${ }^{14}$ la competencia (35.59\%), el desempeño (20.00\%), la capacitación $(38.64 \%)$, la participación $(0.68 \%)$, el sentido de responsabilidad $(7.80 \%)$, los periodos de prueba $(28.81 \%)$ y la puntualidad $(7.46 \%)$;

ii) A pesar del avance que han mostrado las empresas examinadas en la polivalencia de sus trabajadores (72.54\%), la decisión para que el trabajador desempeñe otras tareas o puestos distintos a los habituales alcanzó para los años examinados un nivel muy bajo (25.85\%);

iii) Los cambios de horario o días de trabajo es aún una decisión unilateral en la mayoría de los ССт que se analizaron (70.37\%);

iv) Los cambios en los sistemas de trabajo o maquinaria se deciden de manera unilateral (73.26\%), y

v) La prevención de despidos debido a cambios en los sistemas de trabajo o maquinaria en pocos casos contempla un sistema que implique primero la indemnización de los trabajadores y luego la reubicación de éstos (18.06\%).

A modo de conclusión, se puede decir que la flexibilidad en el uso de la fuerza de trabajo no ha adoptado las formas típicas de la llamada flexibilidad funcional de personal; es decir, la flexibilidad en la aplicación de las sanciones, la movilidad interna, las multihabilidades (polivalencia) y la eliminación del escalafón ciego.

La flexibilidad en las condiciones de trabajo (numérica y funcional) podría entenderse como sistema de relaciones industriales, en la medida en que se está hablando de los valores básicos, las leyes, las instituciones y las prácticas organizacionales que rigen el empleo (Dunlop, 1978), o bien la manera en que los trabajadores participan en la organización de la empresa. Como

\footnotetext{
${ }^{14}$ Es importante mencionar que el llamado escalafón ciego es todavía un mecanismo usado para promover a trabajadores a puestos superiores $(49.83 \%)$.
} 
acertadamente manejan algunos especialistas en la materia, específicamente se trata de la participación de los trabajadores en el diseño del proceso, la división del trabajo y la forma de asignación de tareas, la capacitación, las funciones de los puestos, la estructura jerárquica, los grupos informales de trabajo, las relaciones interpersonales dentro del trabajo, los conflictos entre obreros y de éstos con los mandos medios y la negociación colectiva formal e informal en los centros de trabajo, en cuanto a la interpretación de las normas laborales y la incertidumbre de los trabajadores.

Las principales variables que se utilizaron para tener una visión objetiva del indicador que ahora nos ocupa fueron: la participación del trabajador en el desarrollo de la empresa, los acuerdos explícitos de modernidad productiva y las nuevas formas de relación sindicato-empresa. Los datos obtenidos en la muestra analizada nos dan suficiente evidencia para asegurar que la participación de los trabajadores en la organización de la empresa es un rubro que lejos de avanzar, va rezagándose.

Para 1994 y 2000, dicho indicador presentó una reducción de 7.03 puntos porcentuales, los cuales equivalen a una TMCA negativa de 6.30 por ciento. Por rama de actividad económica, se puede observar una situación semejante, ya que para los años examinados $51.72 \%$ de dichas ramas se ubicaron por debajo de la media general de participación de los trabajadores en la organización del trabajo. Los datos anteriores sugieren que pese a que las empresas han mostrado disponibilidad para modernizar la organización productiva, todavía un gran porcentaje de éstas no han signado pactos bilaterales con los sindicatos en los que se contemple la participación de los trabajadores en los procesos de trabajo. Es importante reconocer que por tamaño de empresa, las clasificadas en el estrato 1 se ubicaron por debajo del promedio general, y las empresas grandes y medianas fueron las únicas en superar dicho promedio. Tentativamente se podría suponer que los Сст de las empresas del estrato 3 fueron firmados en su mayoría por sindicatos aglutinados en centrales obreras de fuerte impacto, ${ }^{15}$ amén de que dichas empresas tienen ciclos de vida más largos.

\footnotetext{
${ }^{15}$ De las empresas clasificadas en el estrato 3, 65\% estaban aglutinadas en la Confederación de Trabajadores de México (Стм) o en la Confederación Revolucionaria de Obreros y Campesinos (CROC). La primera, por ser la más representativa en el ámbito nacional, garantizó, hasta hace muy poco tiempo, un acceso directo al partido gobernante y al sistema nacional de relaciones industriales. Esta situación de integración
} 
Al realizar un examen más riguroso, podemos observar que la mayoría de los ССT analizados no presentan cláusulas que hagan referencia a la existencia de comisiones mixtas de participación de utilidades (71.19\%), del cuadro general de antigüedad (63.73\%), de consulta de la comisión nacional de salarios mínimos (98.99\%), departamentales (98.99\%), de patrimonio de vivienda (98.99\%), de fomento deportivo (98.97\%), de tabuladores de salarios (97.98\%) y otro tipo de comisiones (81.82\%). En la mayoría de los ССТ analizados no se contemplan sistemas participativos entre empresa y sindicato para discutir la organización del trabajo (98.31\%) y para premiar la participación y/o creatividad de los trabajadores (98.98\%). En cuanto a los сст que no contienen dichas cláusulas, se observa que en la mayoría de éstos no se menciona como prevención (99.66\%) ni como objetivo $(100 \%)$ la creación de dichas precisiones. Asimismo, la existencia de convenios de modernidad entre trabajadores y empresa es casi nula en algunos aspectos: productividad (18.98\%), incremento de la calidad (9.15\%), círculos de control de calidad $(0.68 \%)$, cargas de trabajo $(0.68 \%)$, prevención de accidentes $(3.05 \%)$, trabajo en equipo $(0.68 \%)$ y desperdicio de materiales (5.42\%). Asimismo sólo 12 empresas que no han firmado este tipo de convenios, los mencionan como objetivos futuros a cumplir.

Es un hecho que la participación de los trabajadores en la organización productiva no ha mostrado grandes avances en nuestro país debido posiblemente a que los pactos entre los sindicatos y las empresas se han caracterizado por ser meramente defensivos o de la circulación, pues sólo se ocupan de la vigilancia de las condiciones de trabajo que pudieran perjudicar a sus agremiados y no plantean propuestas o alternativas de cómo participar en los cambios tecnológicos gestados desde el interior de las empresas, ${ }^{16}$ amén de que en el proceso de modernización de las condiciones generales de trabajo quedan excluidos los trabajadores. Lo anterior se deriva de que una parte mínima de los ССT examinados (4.07\%) refleja que las funciones de los sindicatos estén relacionadas con el logro de cambios favorables en la organización del trabajo, con la flexibilización de éste, el establecimiento de

vertical y de centralización nacional tiene algunas ventajas, por ejemplo, facilita el establecimiento de consultas y arreglos institucionalizados entre los actores de las relaciones industriales, así como la acción del sindicalismo en calidad de representante de las demandas de los trabajadores como de gestor político del sector (Arciniega, 1999).

${ }^{16}$ Actualmente se observa que muchos sindicatos están perdiendo la capacidad de legitimidad, pese a que su capacidad de expansión sigue avanzando. 
las nuevas condiciones de trabajo y la realización de estudios para fijar los estándares de producción. De igual manera ninguno de los CCT examinados menciona la prevención de funciones ni el tipo de éstas que puede llevar a cabo el sindicato en cuanto a tratar de lograr cambios deseables en la organización del trabajo. De 1994 a 2000 el nivel de organización productiva, de acuerdo con la muestra de СCT, registró una disminución en 1996 de 5.46 puntos porcentuales con respecto a 1995, con lo que se obtiene una TMCA de 0.29 por ciento. Es un hecho que la participación de los trabajadores en la organización productiva ha sido menor que la flexibilidad numérica y la flexibilidad funcional. Lo anterior podría deberse a que los cambios acordados por las partes en las cláusulas de los Сст examinados buscan en general reducir la participación de los sindicatos en cuanto a regulación del contrato, decisiones sobre productividad y calidad; de manera que dicha regulación tiende a la unilateralidad, además de que la flexibilidad laboral deja de tener consenso y la participación de los trabajadores y el involucramiento de los sindicatos se aleja de las decisiones en cuanto se refiere a productividad y calidad en el trabajo.

El grado de organización productiva por rama de actividad económica presenta un comportamiento muy similar al expuesto anteriormente, es decir, sólo 15 ramas de actividad superaron a la media general de organización productiva. Las ramas que obtuvieron el mayor índice de organización productiva fueron: eléctrica, calera, cinematográfica, maderera y vidriera. Lo anterior podría deberse a que estamos hablando de sectores económicamente fuertes en cuanto a inversión de capital variable y que cuentan con grandes inversiones de capital fijo (nacional y/o extranjero), lo que da la posibilidad de poner en práctica programas de reorganización del proceso de trabajo y de la mano de obra sin mermar dicho capital. Asimismo, se trata de empresas cuyo giro comercial está estrechamente vinculado con el uso de nuevas tecnologías para la creación de sus productos y/o servicios. De alguna forma la aseveración anterior nos podría conducir a pensar que, por lo menos algunas empresas grandes, se están incorporando al proceso de flexibilización de las condiciones generales de trabajo que desde la crisis de 1982 vienen implantando los sistemas de relaciones industriales.

En cuanto a los recursos humanos, podemos decir que no sólo son entendidos como el esfuerzo o la actividad humana, sino también comprende los conocimientos, experiencias, motivaciones, intereses personales, aptitudes, actitudes, habilidades, po- 
tencialidades y salud de la mano de obra. La modernización de los sistemas de recursos humanos no sólo beneficia a los empresarios, sino que también da a los trabajadores la capacidad para incrementar sus competencias laborales en la medida en que adquieran mejores atributos para poder competir en el mercado laboral. Así, para evaluar esta dimensión nos debemos remitir a la capacitación y al bienestar de los trabajadores, porque estos indicadores permiten a la empresa crear sistemas de producción más competitivos en los mercados nacionales e internacionales, $\mathrm{y}$ a los trabajadores la posibilidad de elevar su productividad, y por ende su nivel de vida.

La propuesta de la modernización productiva encuentra su punto más álgido en la flexibilización del trabajo y en la automatización, con las cuales la actividad empresarial en las áreas de planeación, producción, investigación y administración quedan simplificadas. Dicha modernización productiva altera también las demandas de educación profesional y técnica, de ahí que actualmente las empresas requieran de técnicos y trabajadores polifuncionales competentes en amplios campos o especialidades (García, 1995; 17) que engranen con las nuevas formas de producir. El acceso a estas habilidades puede ser obtenido a través de la introducción de programas de capacitación que conviertan al trabajador tradicional en un trabajador polivalente que sea capaz de desempeñar de manera óptima las tareas asignadas. Indudablemente la capacitación se ha convertido en la panacea de la flexibilidad de las condiciones generales de trabajo, en tanto que permite eliminar o disminuir la parcelación del trabajo, la desmotivación, el sabotaje, el exceso de desechos y productos defectuosos, disminuir costos y elevar la productividad. Las variables que se utilizan para medir este indicador tienen que ver con:

i) La incorporación de cursos de adiestramiento y readiestramiento de la mano de obra de nuevo ingreso y en activo;

ii) La extensión de los programas de capacitación y la vinculación de éstos con sistemas de promoción a puestos superiores;

iii) Un sistema de rotación de puestos de trabajo;

iv) Los incentivos por la disposición de los trabajadores a capacitarse, $\mathrm{y}$

v) La creación de un sistema integral y permanente de capacitación en los centros de trabajo. 
Los datos obtenidos del informe de la STPS nos permiten observar que la capacitación es un indicador que presentó un avance de 18.02 puntos porcentuales entre 1994 y 2000, a una TMCA de 6.33\%. Por rama de actividad económica, 10 de ellas rebasaron la media general. En cuanto al tipo de estrato, se puede ver que son las empresas medianas y grandes las que están incorporando programas de flexibilidad en cuanto a capacitación se refiere. En la mayoría de los ССT examinados se mencionan cláusulas que hagan referencia:

i) A la capacitación de los trabajadores en $98.98 \%$;

ii) Cursos de adiestramiento para los trabajadores de nuevo ingreso en $96.04 \%$;

iii) Cursos de readiestramiento de la mano de obra en activo en $96.21 \%$;

iv) Cursos de capacitación para la promoción de puestos superiores en $96.13 \%$, y

v) Extensión de los cursos de capacitación a todas las áreas en $97.83 \%$.

En términos generales, se puede plantear que la mayoría de las empresas examinadas depositó en la JFCA revisiones contractuales en donde la capacitación aparece como un requisito legal que estipula la LFT y que obviamente tiene carácter de obligatoriedad, de ahí que la mayoría de dichas empresas no estipulen en sus CCT cláusulas que permitan observar al 100\% prácticas de modernidad en las condiciones de trabajo o que hagan referencia a la capacitación (por ejemplo, el otorgamiento de incentivos a los trabajadores por participar en los cursos de capacitación, sistemas instituidos de rotación de puestos para el aprendizaje de diferentes tareas, mención de objetivos para la creación de dicho sistema, prevención de la capacitación formal, sistemas integrales de capacitación permanente, etcétera).

En cuanto al bienestar social de los trabajadores, se puede decir que se trata de un indicador que también permite analizar los recursos humanos con los que cuentan las empresas. Este rubro hace referencia al conjunto de elementos que están relacionados con el propósito de mejorar la retribución del factor trabajo y que, en gran medida, tienden a mejorar el poder adquisitivo de los trabajadores. Las variables que permiten medir este indicador están referidas, por un lado, a los riesgos que asumen los trabajadores en el proceso de trabajo y a las compensaciones que obtienen éstos por asumir tales riesgos, y por otro, a las presta- 
ciones y remuneraciones que recibe la mano de obra por la venta de su fuerza de trabajo. Los resultados que obtuvo la STPS nos dan evidencia para suponer que el bienestar de los trabajadores es un tema sobre el que han insistido muchas empresas, es decir, que en la contratación colectiva se observan casi siempre cláusulas que reconocen que la salud es un recurso con que cuentan los miembros de la organización y que no sólo se refiere a la ausencia de enfermedad, sino además a un estado completo de bienestar físico, mental y laboral, aunque hay que mencionar que el monto asignado a estas prestaciones aún es bajo.

La afirmación anterior puede sustentarse en los resultados que se obtuvieron en este rubro. El crecimiento del bienestar de los trabajadores entre 1994 y 2000 alcanzó 10.97 puntos porcentuales a una TMCA de 3.94\% y en donde 15 ramas de actividad superaron la media general. En cuanto al tamaño de empresa, los resultados siguen indicando que las empresas medianas y grandes son las que en sus ССТ estipulan cláusulas que hacen referencia a programas integrales de bienestar social para sus trabajadores. De los resultados obtenidos de los ССт muestreados se puede observar lo siguiente:

i) La mayoría de las empresas menciona políticas de prevención para abatir los riesgos de trabajo (95.93\%), y otorga equipo de seguridad a los trabajadores (65.76\%); en cambio, no se hace referencia a la existencia de un sistema para abatir dichos riesgos $(100.00 \%)$;

ii) Sólo en pocos casos excede a lo estipulado en la LFT: los días otorgados a los trabajadores por concepto de vacaciones al primer año de labores (42.03\%), los beneficios que reciben los familiares de un trabajador en caso de que éste fallezca $(22.37 \%)$, el pago de las incapacidades permanentes totales (4.75\%) y temporales (1.02\%), aunque es importante reconocer que para el caso de los días otorgados por aguinaldo en la mayoría de los contratos se observa que dichos días superan lo que se estipula en la ley $(79.32 \%)$;

iii) La ayuda para renta de casa (4.41\%), ayuda para becas (34.24\%), transporte $(27.12 \%)$, servicios médicos especiales (24.75\%), ayuda para comprar lo que la empresa produce (7.46\%), préstamos (23.39\%), y el fondo adicional para la jubilación $(8.81 \%)$ todavía son poco significativos en cuanto a las condiciones de trabajo que hacen referencia al mejoramiento del bienestar de los trabajadores; 
iv) La puesta en marcha de sistemas de remuneraciones adicionales al salario ligado al cumplimiento de metas que tengan que ver con la producción y/o servicio (29.83\%), calidad (4.41\%), capacitación (1.69\%), el nivel de ventas (4.07\%), utilidades (8.81\%), puntualidad (36.95\%), asistencia (40.68\%) y con alguna comisión (8.81\%) no es una tarea prioritaria;

v) El salario diario menor aún es igual o menor a dos salarios mínimos (88.82\%);

vi) El número de niveles salariales existentes en los tabuladores de los Сст sigue estando por debajo de 10 (56.61\%) y el número de categorías de puestos de trabajo existentes en éstos es menor de 21 (71.53\%), y

vii) El salario diario mayor y la media salarial diaria están por debajo de los seis salarios mínimos (80.34\% y 94.92\%).

Esta dimensión metodológica presentó resultados más alentadores que los que hacen referencia a la organización productiva. En el periodo de 1994 a 2000, la dimensión denominada recursos humanos creció 14.49 puntos porcentuales a una TMCA de $5.15 \%$. Por tamaño de empresa, sólo las que tienen más de 100 trabajadores rebasaron la media general. Por rama de actividad económica, 12 de éstas rebasaron la media salarial promedio correspondiente. Es un hecho que estamos hablando de ramas de actividad sin fuertes rezagos económicos y que su giro comercial está estrechamente vinculado con el uso de nuevas tecnologías para la creación de sus productos y/o servicios. ${ }^{17}$

\section{Conclusiones}

Al realizar un balance final es posible decir que si bien existen algunas áreas en las que las empresas examinadas han puesto a funcionar sistemas para la modernización de sus procesos de trabajo, también es cierto que a la mayoría de éstas les falta mucho camino por andar. Políticas que contengan como objetivos la flexibilidad numérica, funcional, participación de los trabajadores en la organización productiva y capacitación de éstos, casi no están estipuladas en las cláusulas que componen los СCT analizados. Es evidente que las decisiones bilaterales entre el sindicato y la empresa se notan más en el bienestar de los trabajadores, de ahí que el proceso de flexibilización de las condiciones generales de trabajo, al menos en las empresas observadas, aún sea lento.

${ }^{17}$ Eléctrica, calera y cinematográfica. 
Se puede decir que en el periodo de 1994 a 2000 el grado de flexibilidad en las condiciones generales de trabajo creció apenas 6.17 puntos porcentuales, lo que equivale a una TMCA de $2.57 \%$. Es claro que la flexibilización de las condiciones generales de trabajo es casi exclusiva de las empresas que:

i) Cuentan con un gran número de trabajadores;

ii) Su giro comercial está ligado a la instrumentación e innovación tecnológica ${ }^{18}$ (telefonía, educación, transporte aéreo, metalúrgica, siderúrgica, petroquímica, automotriz y banca);

iii) Cuentan con grandes niveles de capital;

iv) Con inversiones de origen extranjero;

v) Territorialmente se ubican en las entidades federativas más industrializadas (Distrito Federal, Guadalajara, Monterrey, Sonora, Tijuana y Puebla);

vi) Están agrupadas en sindicatos poco defensivos, y

vii) Tienen establecimientos en más de una entidad federativa y que son las más protectoras.

Se puede plantear como hipótesis que en México los procesos de reestructuración productiva y, particularmente, de la flexibilización de las condiciones generales de trabajo, se han caracterizado por formas específicas de resistencia sindical, por la falta de proyectos laborales que hagan frente a las nuevas formas de organización del trabajo y por la negativa de las gerencias a que sus trabajadores desempeñen funciones que rebasen el trabajo mecánico y rutinario. No se trata de que la automatización flexible alcance a todas las áreas y niveles de las empresas, sino de ajustar aquellas que aún encuentran apoyo en las máquinas-herramientas a los nuevos procesos de trabajo. El reto que enfrenta el aparato productivo mexicano es todavía la flexibilidad de las condiciones generales de trabajo, vía por ejemplo la flexibilidad laboral, que traería como beneficios:

i) Un mejor aprovechamiento de la inversión en equipos y mano de obra con la consecuente reducción de tiempos muertos;

ii) El aumento de la eficacia productiva a través de la incorporación de equipos productivos;

iii) Reducción de inventarios;

iv) Series cortas de producción de acuerdo con el producto y la materia prima, generando así el incremento de la producción de acuerdo con las variaciones del mercado;

${ }^{18}$ Por rama de actividad, 15 de éstas rebasaron $48.61 \%$ obtenido como promedio. 
v) Trabajadores polivalentes que se traducirían en menores costos de capital variable para los empresarios, y finalmente

vi) Una sustancial mejora para los trabajadores en cuanto a niveles de vida y productividad.

Si bien es un hecho que las políticas del Estado neoliberal orientadas a las nuevas circunstancias de apertura de los mercados y de la globalización implicaron la presencia de empresas modernas y competitivas, también es un hecho que los procesos de flexibilidad laboral no sólo deben entenderse como un problema técnico para optimizar las funciones de producción, en tanto que las repercusiones de dichos procesos afectan a los sistemas de relaciones industriales en su conjunto. De manera que lo anterior implicó que, por lo menos en la muestra examinada, en dicho proceso se dieran luchas y negociaciones entre el Estado, las gerencias y los sindicatos, aunque al final estos últimos se convirtieron en aliados del Estado y aceptaron sin ninguna objeción las políticas de flexibilidad laboral a cambios de algunos privilegios y beneficios. Indudablemente los sindicatos tenían la responsabilidad de que el modelo neoliberal funcionara a través de velar por el control obrero ante los continuos ajustes macroeconómicos para disminuir los índices de inflación. Lo anterior implicó que los trabajadores ya no recibieron por parte del Estado incrementos salariales reales, sino que sus remuneraciones estuvieron vinculadas en el plano microeconómico, es decir, a través de la difusión de los pactos por productividad. De alguna forma, las remuneraciones vía bonos de productividad implicaron una contradicción sui generis entre libre mercado y participación sindical. Indudablemente el modelo neoliberal nunca contempló coyunturas económicas difíciles como la que se presentó en diciembre de 1994, ni la existencia de un abanico de posibilidades de estrategias microempresariales que tenían que ver con distintos tipos de cultura laboral, así como de sindicatos, trabajadores y empresas.

En esta tesitura, las organizaciones sindicales que apoyaron y se beneficiaron de las políticas del Estado benefactor deberán ser modificadas a favor de la reestructuración económica que viene experimentando México a partir de la década de los años ochenta. Aunque hay que aclarar que las políticas neoliberales no son las únicas alternativas de modernización laboral, en tanto que es poco juicioso argumentar que la flexibilidad laboral se lleve a cabo en todas las empresas y que los sindicatos ya no 
tengan tareas que cumplir. Asimismo, es importante mencionar que las políticas neoliberales pocas veces tuvieron como referente los pisos de trabajo, de manera que el Estado mostró un radicalismo de flexibilidad laboral poco aplicable, en tanto que suponía un sistema de multitareas indiscriminado y extrema inseguridad en el trabajo que contrariaba la identidad de los trabajadores. Lo anterior nos lleva a pensar que el proceso de flexibilización laboral en México nunca ha sido ni será homogéneo, en tanto que se trata de un espacio en construcción social.

Asimismo, y pese a que el grado de flexibilización de los CCT examinados no es muy alto, posiblemente porque los aspectos codificados en la LFT algunas veces limitan que dicho proceso siga su curso, se podría pensar que en la práctica se den relaciones laborales más flexibles. Se observa que el proceso de flexibilidad laboral ha involucrado a muy pocas empresas, específicamente a aquéllas que fueron privatizadas antes de su venta para sanear la economía y que conformaban el núcleo duro del patrón contractual de la Revolución Mexicana.

Se puede asegurar que para las empresas mexicanas la flexibilidad laboral sigue un proceso lento. Tentativamente se podría sugerir que lo anterior se debió en gran parte a que los acuerdos que implantó el Estado mexicano, las cúpulas empresariales y sindicales sobre flexibilidad, fueron entendidos por las dos últimas como acuerdos con carácter de obligatoriedad, que orillaron a muchos sindicatos y empresarios a signar contratos de protección que se limitaron a aplicar con base en lo estipulado por la LFT y comúnmente a reflejar los acuerdos sobre flexibilidad laboral en ССт de formato único y válido, por lo menos para las empresas agremiadas al mismo sindicato, central obrera o aglutinadas en una rama de actividad económica. ${ }^{19}$ Indudablemente el aspecto más urgente de la flexibilización en las condiciones generales de trabajo es la lucha contra esa realidad extralegal de los

\footnotetext{
${ }^{19} \mathrm{El}$ procedimiento de contratos de protección evita que proceda un auténtico reclamo de firma de Сст, у es un procedimiento que beneficia a las cúpulas sindicales corporativas que administran dichos contratos, en tanto que éstas reciben los beneficios políticos y económicos por la representación que ostentan y el pago de cuotas de las que generalmente no rinden cuentas. El derecho de asociación en sindicato, la contratación colectiva misma y el supuesto clima de tranquilidad que priva en las relaciones laborales son consecuencia de la inexistente vida sindical en México y la falta de una real contratación colectiva. Fueron tales los alcances de los contratos de protección, que algunas veces desde antes de que se inaugurara algún establecimiento, ya se contaba con un СCT firmado y depositado en la JFCA. En términos generales, los contratos de protección caracterizaron la contratación colectiva en México durante mucho tiempo provocando una cultura de negociación laboral sustentada en la representación corporativa de obreros y empresarios.
} 
llamados contratos de protección, que en la realidad son contratos fantasmas pactados en el ámbito cupular sin el conocimiento ni consentimiento de los trabajadores.

De acuerdo con el análisis realizado, se cree que el proceso de flexibilidad productiva seguirá siendo en México un debate sobre el que se debe insistir, particularmente sobre la resistencia sindical y la actuación cada vez más conservadora de los sindicatos en las formas de negociación laboral, así como en la capacidad que deben tener los empresarios para rediseñar proyectos alternativos que hagan frente a tal proceso. Proyectos que reconozcan que la aplicación de formas flexibles de producción podría ser una de las salidas a la crisis del modelo de acumulación fordista y, específicamente, a la caída de las tasas de productividad. Se trata de sustentar la flexibilidad laboral en el abandono de formas verticales de producción, lo que puede traer como beneficios un mejor uso de la fuerza de trabajo, mejores estándares de calificación, niveles salariales estables y menores costos laborales acompañados de mejores condiciones de trabajo. Por lo anterior se sugiere que los estudios sobre flexibilidad laboral no deberán dejar a los automatismos del mercado el problema del futuro de la contratación colectiva, sino que deben partir del supuesto de que los trabajadores son actores sociales que toman decisiones y que de éstas también dependerán las formas de negociación colectiva que predominarán en los próximos años. Lo anterior nos conduce a pensar, no en términos de flexibilidad productiva, sino de horizontalidad laboral, en tanto que la contratación colectiva depende de: procesos sociales y políticos; de las gestiones que llevan a cabo los representantes sindicales; de los vínculos establecidos entre los trabajadores y los líderes sindicales; de la resistencia de las gerencias para impulsar programas de flexibilidad laboral y de productividad, y del papel que juegan las instituciones públicas en la firma de los СCT. De igual modo, dichos estudios deberán tener en cuenta que pese a que los CCT todavía presentan gran énfasis en la parte salarial, es obvio que éstos tienen cada día un papel más amplio en la definición de las formas de organización de trabajo.

Evidentemente son muchos los requisitos que las empresas mexicanas deberán cumplir para alcanzar un grado de flexibilidad laboral satisfactorio que les permita competir en los mercados internacionales; se habla entonces de la necesidad de desarrollar una estrategia adecuada para aprovechar las características naturales y sociales de México, que modifiquen las antiguas formas de acumulación, pensando que los cambios políticos y socia- 
les no pueden darse sólo porque los líderes sindicales lo decidan. El reto es el diseño de un nuevo patrón de acumulación que combine las nuevas tecnologías con la capacidad productiva. Para lograrlo es indispensable que el Estado, los empresarios, las organizaciones sindicales y los trabajadores adquieran un papel decisivo en la organización y planificación en el proceso de flexibilización laboral, tema central de este artículo.

\section{Bibliografía}

Arciniega, Rosa (1999), "Condiciones de trabajo en la industria textil: el caso de la industria del valle de Toluca-Lerma", en Cambios en las Relaciones Laborales, vol. 2, UAM, México.

Bouzas, José Alfonso y Enrique de la Garza (1999), "El cambio en la contratación colectiva de jurisdicción federal y local", en Enrique de la Garza y Alfonso Bouzas (coords.), Cambio en las relaciones laborales en México. Enfoque sectorial y regional, vol. 1, UAM, México.

Castilla, B. y B. Torres (1999), "Las relaciones laborales en Yucatán”, en Enrique de la Garza y José Alfonso Bouzas (coords.), Cambio en las relaciones laborales en México. Enfoque sectorial y regional, vol. 2, UAM, México.

De la Garza, Enrique (comp.) (1999), "Modelos de industrialización en México", en Cuaderno del Trabajo, núm. 15, sTPS, México.

Dunlop, J. (1978), Sistema de relaciones industriales, Barcelona, Península.

García, C. (1995), Capacitación y reestructuración productiva en América Latina, UAM, México.

González, Sergio (1999), "Reestructuración industrial y cambio en las relaciones laborales en el Estado de México", en Enrique de la Garza y José Alfonso Bouzas (coords.), Cambio en las relaciones laborales en México. Enfoque sectorial y regional, vol. 2, UAM, México. 
Meyer, Gerald (1989), Dualistic development, Oxford University Press, us.

Remmling, W. Gunter (1990), "La realidad social según la teoría de occidente", en Tiempo, realidad social y conocimiento, Siglo XXI, México.

Trouve, P. (1989), “¿Management de las flexibilidades o flexibilidades del management?”, en Revista Sociología del Trabajo, núm. 4, Siglo XXI, Madrid.

Wong, Pablo (1996), Las regiones en la globalización, Hermosillo, El Colegio de Sonora, México.

Zapata, Francisco (1995), El sindicalismo mexicano frente a la reestructuración, colmex, México.

Enviado: 8 de noviembre de 2001 Aceptado: 23 de enero de 2002 